\title{
Mycoplasma pneumoniae-associated Severe Fatal Autoimmune Hemolytic Anemia: A Rare Entity
}

\author{
Mahesh Mishra ${ }^{1}$, Vikram K Jain ${ }^{2}$, Ram M Jayaswal ${ }^{3}$
}

\begin{abstract}
Mycoplasma is the smallest living-free organism in the nature. It is an important and common cause of respiratory infections. It mainly affects children more than 5 years of age and younger adult causing disease of varied severity of illness from asymptomatic or upper respiratory infection to severe pneumonias. Hemolytic anemia is known as a rare but severe complication of Mycoplasma infection. We encountered a 15-year-old male with cold agglutinin antibody-associated fatal autoimmune hemolytic anemia secondary to Mycoplasma pneumonia hence reported.

Keywords: pneumonias, hemolytic, Mycoplasma.

Journal of Mahatma Gandhi University of Medical Sciences \& Technology (2018): 10.5005/jp-journals-10057-0089
\end{abstract}

\section{INTRODUCTION}

Mycoplasma is the smallest living-free organism in the nature. It is an important and common cause of respiratory infections. It mainly affects children more than 5 years of age and younger adult causing disease of varied severity of illness from asymptomatic or upper respiratory infection to severe pneumonias. About $25 \%$ of Mycoplasma infections are associated with an extrapulmonary complication at variable time. These may occur even in the absence of pulmonary symptoms. Any system of body may have disease manifestations. Common are central nervous system (CNS), cardiac, dermatologic, gastrointestinal musculoskeletal, and hematological. Hemolytic anemia is known as a rare but severe complication of Mycoplasma infection. Occasional case reports of severe hemolytic anemia are available in the literature. ${ }^{1-4}$ We are not able to find any Indian case report of Mycoplasma pneumonia causing fatal severe hemolytic anemia. We encountered a 15-year-old male with cold agglutinin antibody-associated fatal autoimmune hemolytic anemia secondary to Mycoplasma pneumonia hence reported.

\section{Case Description}

A 15-year-old male was admitted to our hospital for pyrexia of high-grade, cough with expectoration and breathlessness since 15 days. Patient was asymptomatic couple of weeks before he had cough along with fever and rapidly progressing breathlessness. There was no significant past history of similar illness. A family history was not contributory to present illness. Patient was put on Coamoxiclav with artesunate by local practitioner without any relief. On general physical examination, patient was conscious, oriented to time, place, and person. His blood pressure was 100/60 mm Hg and pulse rate was 140 minutes. Respiratory rate was $24 /$ minute with the use of accessory muscles of respiration. Oxygen saturation was $90 \%$ on room air. Patient was febrile and pallor. There was no cyanosis, lymphadenopathy clubbing, and pedal edema. Respiratory system examination revealed bronchial breathing with crepts in the right infrascapular area. Other system examinations were unremarkable.

On admission, chest X-ray showed right middle and lower zone opacities (Fig. 1); an arterial blood gas analysis indicated type I respiratory failure. Hematological investigation showed

\begin{abstract}
${ }^{1-3}$ Department of Respiratory Medicine, Mahatma Gandhi Medical College and Hospital, Mahatma Gandhi University of Medical Sciences and Technology, Jaipur, Rajasthan, India

Corresponding Author: Mahesh Mishra, Department of Respiratory Medicine, Mahatma Gandhi Medical College and Hospital, Mahatma Gandhi University of Medical Sciences and Technology, Jaipur, Rajasthan, India, Phone: +91 9414460198, e-mail: maheshmishradr@ gmail.com

How to cite this article: Mishra M, Jain VK, Jayaswal RM. Mycoplasma pneumoniae-associated Severe Fatal Autoimmune Hemolytic Anemia: A Rare Entity. J Mahatma Gandhi Univ Med Sci Tech 2018;3(3):113-115. Source of support: Nil

Conflict of interest: None
\end{abstract}

hemoglobin of $2.3 \mathrm{~g} / \mathrm{dL}$ with a mean corpuscular volume of $91 \mathrm{~mm}^{3}$. Reticulocyte count was $12 \%$ with lactate dehydrogenase (LDH) of 1,000 and total leukocyte count of $64,315 / \mathrm{mm}$ with $68 \%$ polymorphs. Platelets were $800,000 / \mathrm{mm}^{3}$. Peripheral blood film had band form, metamyelocyte, and occasional nucleated red blood cell. Blood sugar, urea, creatine, electrolyte, and routine urine

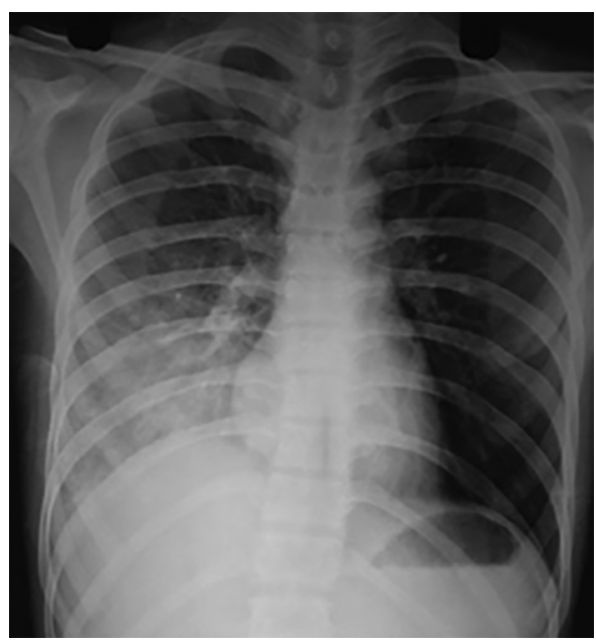

Fig. 1: Chest radiograph (posteroanterior view) showing right lower zone opacity 


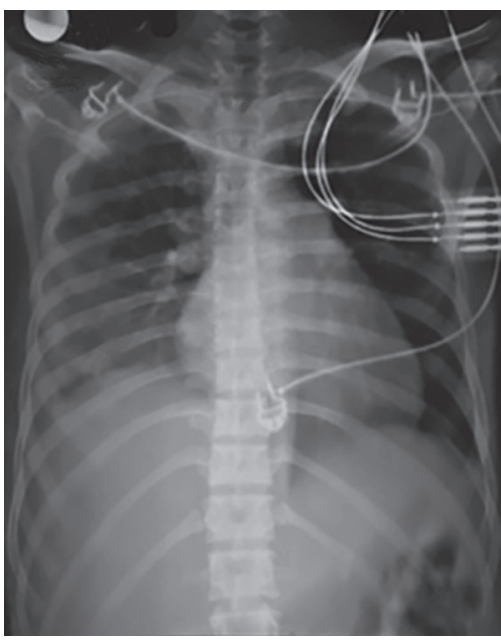

Fig. 2: Chest radiograph (posteroanterior view) after 2 weeks showing resolution of right lower zone opacity

microscopic examination were within normal limits. Liver function was abnormal with level of total bilirubin of 3 in which 1.7 was of indirect, serum glutamic-oxaloacetic transaminase (SGOT) was 87 while serum glutamate-pyruvate transaminase (SGPT) was within normal limits. Serum protein was $5.1 \mathrm{~g}$ with albumin level of 2.7. Prothrombin time was normal. Antigen test for malaria test was negative. Serology for dengue and scrub typhus was negative. Blood culture was sterile. Sputum for acid fast bacilli, gram stain, and pyogenic culture remained nonconclusive. Ultrasonography of abdomen was normal. Direct Coomb test was positive. Glucose 6 phosphates, serum vitamin $B_{12}$, and hemoglobin electrophoresis were within normal limits; pathologist gave us important input that red blood cells were agglutinating at colder temperature while deagglutinated on warming at $37^{\circ} \mathrm{C}$. Cold agglutinin antibody was positive. IgG and IgM for Mycoplasma were strongly positive as their serum values were 28.33 and 1.97 , respectively, by enzyme immunoassay (EIA) method.

Patient was put on iv methylprednisolone along with azithromycin as supportive treatment with warmer placement aside patient to avoid cold. Blood transfusion was planned as the patient had very severe hemolysis. But we were not able to execute it, as the blood group was not identifiable due to autoagglutination of red blood cell (Fig. 2). Considering life-threatening emergency, we tried to infuse $\mathrm{O}$ negative blood as it is a universal donor under the supervision of hematologist but did not succeed because of hemolytic reactions during transfusion. Consolidation began to resolve (Fig. 2), but hemolytic reactions were persisting. Possibility of plasmapheresis was also discussed but not executed because of very low level of hemoglobin. So, intravenous rituximab was administered at a dose of $375 \mu \mathrm{g} / \mathrm{m}$ body surface area. Patient continues to deteriorate requiring mechanical ventilation ultimately succumb to death despite of our best effort (Fig. 3).

\section{Discussion}

Autoimmune hemolytic anemia consists of warm and cold antibodies that are directed against antigen against red blood cell surface. These antibodies may be primary or secondary to any underlying cause like infections, malignancy, etc. Cold agglutinin disease is rare contributing $15 \%$ of autoimmune hemolytic anemia with incidence of 1 per million every year. ${ }^{5}$ Cold agglutinin titer is

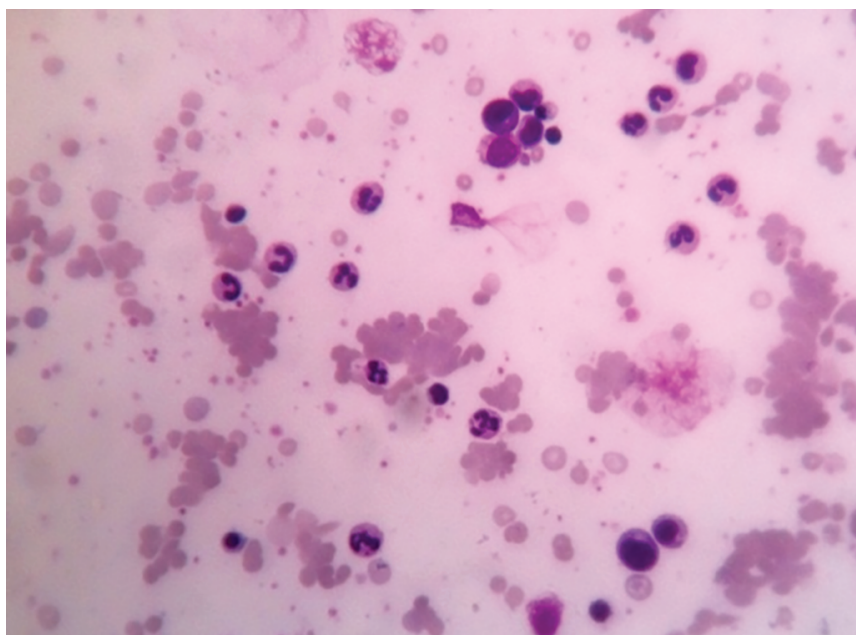

Fig. 3: Peripheral blood film showing red cell agglutination

frequently observed during Mycoplasma infection. About 50\% of patients infected with Mycoplasma have cold agglutinin. ${ }^{6}$ They often result in mild subclinical hemolysis and remain unrecognized. Fatal and severe hemolysis is very rare and often associated with significant lower respiratory tract involvement ${ }^{2}$ as our patient had.

Although Mycoplasma pneumoniae-associated cold agglutinin antibody hemolytic anemia is known as autoimmune disorder, its underlying mechanism is not fully understood. It may involve cross reactions with cold agglutinin antibody, and these antibodies are cross reactive. Autoantibodies develop against human red blood cell against I antigen during the course of Mycoplasma infection. In another hypothesis, they develop directly as a result of antigenic alteration of erythrocyte caused by Mycoplasma pneumoniae Destruction of red blood cell is primarily complement-mediated cascade either directly or immune adherence mediated by targetbound components. Both these processes are common in winters. ${ }^{4}$

Clinical features are nonspecific like our patient. Classical laboratory features are similar to any form of hemolysis. Elevated lactate dehydrogenase and bilirubin levels with increased reticulocyte count were common in cold agglutinin hemolytic anemia. Our patient had hemoglobin level of $2.3 \mathrm{~g}$ only. Such severe hemolysis caused by Mycoplasma pneumoniae has not been reported across the globe as per available literature. Total leukocyte counts may raise suspicion of hematological malignancy as it was in our case. It is similar to case reported by Khan and Yasin. ${ }^{4}$

Clue to diagnosis is autoagglutination of red blood cells on colder temperature which disappears at warm temperature, as was present in our case.

Mycoplasma culture was rarely used for routine diagnosis because of many limitations like nature of organism, incubation period, expensive and specialized growth media, etc. Therefore, diagnosis is usually confirmed by serological test or polymerase chain reaction gene amplification technique. In our patient, diagnosis was based on positive serology and Coomb test in the presence of cold agglutinin antibody similar to other cases reported..$^{1-4}$

Mycoplasma pneumoniae-associated cold antibody hemolytic anemia (CAHA) is usually self-limiting, and most patients recover with conservative treatment. Keeping the patient warm and treatment of underlying condition are the mainstay of treatment. Although antibiotic is likely to have limited value in Mycoplasma 
pneumoniae-associated hemolytic anemia, it is considered immune mediated. But treatment of underlying Mycoplasma infection fastens recovery in the reported case. ${ }^{2-5}$ Unfortunately, our patient continued to deteriorate despite radiological resolution of disease.

In autoimmune hemolytic anemia, blood transfusion can aggravate hemolysis, so this should be used sparingly. If blood transfusion was needed as life-saving measure in case of severe hemolysis or cardiorespiratory compromise, risk of transfusion-related hemolysis may be reduced by keeping warm blood and patient. Blood was successfully transfused in previously reported cases. ${ }^{2,4}$ We did not succeed transfusion in our case because it had most severe hemolytic reactions among reported cases.

Most appropriate pharmacotherapy for CAHA has remained area of clinical research. A favorable response to steroid is present in available few case reports. ${ }^{1,2}$ In a study by Swiecicki et al., around $40 \%$ of patients had response to steroid containing regimen. ${ }^{5}$ Our patient did not respond to steroids.

Rituximab monotherapy and combination therapy with purine analog or azathioprine was analyzed by Swiecicki et al. The response rate was $83 \%$ in single-agent therapy and $79 \%$ in combination therapy. Dose of rituximab is $375 \mu \mathrm{g} / \mathrm{m}^{2}$ weekly for 4 weeks. ${ }^{5}$ Our patient did not give enough time to rituximab response as it rapidly succumbed to disease.

\section{Conclusion}

Our case demonstrates that severe hemolysis caused by Mycoplasma pneumoniae can be fatal even in the absence of other system involvements. This manuscript not only makes a reader aware of rare complications of Mycoplasma pneumonia but also provides insight of its management.

\section{References}

1. Shrestha C, Liu M, Mo Z. Cold agglutinin disease associated with mycoplasma infection in an individual with type 2 diabetes: an atypical case. J Diabet Mellit 2012;2(4):402-405.

2. van der Hoek J, Levin MD, van der Valk PHM, et al. Severe autoimmune haemolytic anaemia in two patients:remember mycoplasma pneumoniae infection. Neth J Crit Care 2008;12(4):167-169. DOI: 10.1186/cc6939.

3. Kurugol Z, Onen SS, Koturoglu G. Severe hemolytic anemia associated with mild pneumonia caused by mycoplasma pneumonia. Case Rep Med 2012;2012:649850. DOI: 10.1155/2012/649850.

4. Khan FY, Yasin MA. Mycoplasma pneumonia associated severe autoimmune hemolytic anemia: case report and review literature. Braz J Infect Dis 2009;13(1):77-79. DOI: 10.1590/s1413-86702009000100018.

5. Swiecicki PL, Hergova LT, Gertz MA. Cold agglutinin disease. Blood 2013;122(7):1114-1121. DOI: 10.1182/blood-2013-02-474437.

6. Gupta R, Gupta A, Goyal V, et al. Mycoplasma pneumonia associated with rhabdomyolysis and guilen bari syndrome case report. Indian J Chest Dis Allied Sci 2005;47(4):305-308. 